\title{
MOLECULAR DOCKING STUDY OF NOVEL COVID-19 PROTEASE WITH CURRENT CLINICAL MANAGEMENT AGENTS
}

\author{
PANKAJ WADHWA* \\ Department of Pharmaceutical Chemistry, Lovely School of Pharmaceutical Sciences, Lovely Professional University, Phagwara, Punjab, \\ India. Email: pankaj.23400@lpu.co.in
}

Received: 29 May 2020, Revised and Accepted: 01 July 2020

\section{ABSTRACT}

Objective: The first case of a new strain of coronavirus (CoV), usually known as CoV disease (COVID)-19, was recognized in Wuhan city of China, in December 2019. Till today, there are no specific treatments available against COVID. During literature searching, it was observed that drugs such as remdesivir, hydroxychloroquine, and chloroquine as their therapeutic options to stop the progress of COVID-19 infections. In the present study, the molecular docking study was performed to understand the binding pattern of selected drugs.

Methods: Molecular docking methods were carried out using molecular virtual Docker software using COVID-19 protease (PDB ID 6LU7), and interactions of these three drugs were visualized.

Conclusion: All three drugs have shown binding interactions with an active site. We assume that these inhibitory activities helped us to identify the possible drug mechanism and further designing of new molecules or investigate the potential use of other available drugs.

Keywords: Coronavirus, Chloroquine, Hydroxychloroquine, Molecular docking, Protease, Remdesivir.

(C) 2020 The Authors. Published by Innovare Academic Sciences Pvt Ltd. This is an open access article under the CC BY license (http://creativecommons. org/licenses/by/4. 0/) DOI: http://dx.doi.org/10.22159/ajpcr.2020.v13i9.38501

\section{INTRODUCTION}

In the last month of 2019 , the first case of a new type of coronavirus (CoV), which is now known as severe acute respiratory syndrome (SARS)-CoV-2, was identified and reported in the Wuhan City of China. Later on, in Guangdong state of China, its human-to-human transmission was first confirmed [1]. After this incidence, in the very first week of March 2020, the World Health Organization has announced it as a global public health emergency. According to reports, this deadly virus has infected almost 210 countries and territories worldwide. The total number of confirmed CoV disease (COVID)-19 infections is more than 2.8 million, and around 1.93 lakh (it is equal to 0.193 million) death occurred in the whole world. It was observed that around $3 \%$ of cases are more serious and the virus is spreading at a rapid rate through air distance, airborne transmission, duration on objects, and surfaces, respectively [2]. However, in the meanwhile, the various drug discovery organizations and academics are continuously working on the development of suitable vaccine and other therapies to effectively treat this epidemic and diminish death cases. In this direction, the crystal structure of the COVID-19 main protease in complex with a peptidomimetic inhibitor (PDB code 6LU7) was developed [3,4]. In many reports, it was studied that CoVs are the members of the retrovirus family and contain its genomic data in form of a long RNA strand. This genome works as an RNA virus, and after infecting the cells, it produces two long polyproteins that are further used in its replication process. These proteins contain a replication/transcription complex which is responsible for synthesizing new virions, and two proteases, respectively. The proteases are well known for their actions. They act through cutting the polyproteins into their functional pieces [5]. Here, in present study, we have performed the molecular docking study of three drugs, such as remdesivir, hydroxychloroquine, and chloroquine against COVID-19 infections. One of the major reasons behind the selection of these three drugs is the ability of remdesivir to inhibit viral replication through termination of RNA transcription. It has also expressed its action against SARS-CoV-2 in vitro methods along with inhibition of activities of other similar beta-CoVs. Apart from this, both hydroxychloroquine and chloroquine are under investigation in clinical trials for pre-exposure or post-exposure prophylaxis of SARS$\mathrm{CoV}-2$ infection. In India, the drug hydroxychloroquine is approved by the Indian Council of Medical Research for the treatment of COVID-19 patients $[6,7]$. These three drugs are well known for their anti-viral and anti-malarial activities, respectively. Their structures, along with their Expanded Lipinski's rule properties, are shown in Table 1 [8]. Aiming to give my contribution, I have performed molecular docking studies of these drugs as protease inhibitors, and their results will help us understand the interactions of these used drugs with the active site of a protein and also help us to design the new molecules with minimal side effects.

\section{METHODS}

\section{Molecular structure and optimization}

The structure of these three drugs, namely, remdesivir, hydroxychloroquine, and chloroquine was drawn by Chem Draw Professionals v15.0.0.106. The geometrical isomers structure of these drugs was further optimized by Chem Bio Draw three-dimensional (3D) Ultra v12.0. All energy minimized structures are saved as in pdb format.

\section{Protein preparation}

To identify desired the protein, an extensive literature survey was carried out using the PubChem database. On the basis of the obtained information, the desired protein was identified and downloaded from the PDB database (https://www.rcsb.org/). The PDB ID 6LU7 was used for our study, and necessary protein preparation steps were carried out using the Molegro Virtual Docker software [9]. The covalently bound peptidomimetic ligand was isolated, and docking was performed in the absent of water molecules. Simulations performed with and without ligand binding to explain the role of COVID-19 protease of the main content of these three drugs. These algorithms utilize a cavity detection algorithm (cavity) to identify the active region to bind of drug (ligand). Screening of the most stable form of ligand combined with MM2 as energy minimizes to get conformational search for generating ligand poses consistent in the active form of the receptor. 

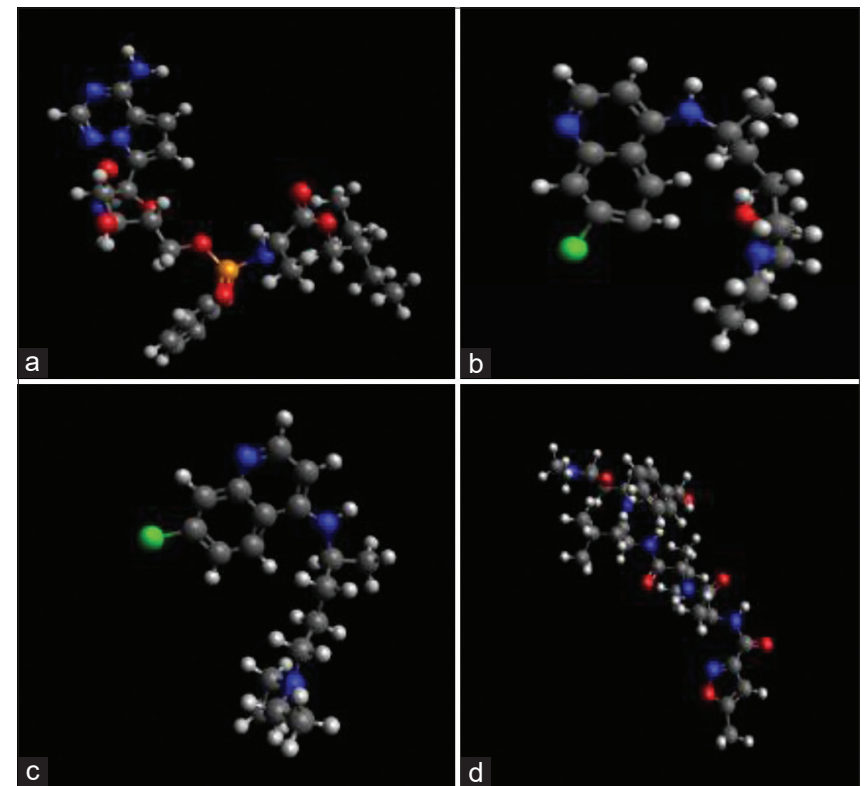

Fig. 1: Three-dimensional structures of selected drugs and cocrystallized ligand (a) remdesivir, (b) hydroxychloroquine, (c) chloroquine, and (d) cocrystallized ligand

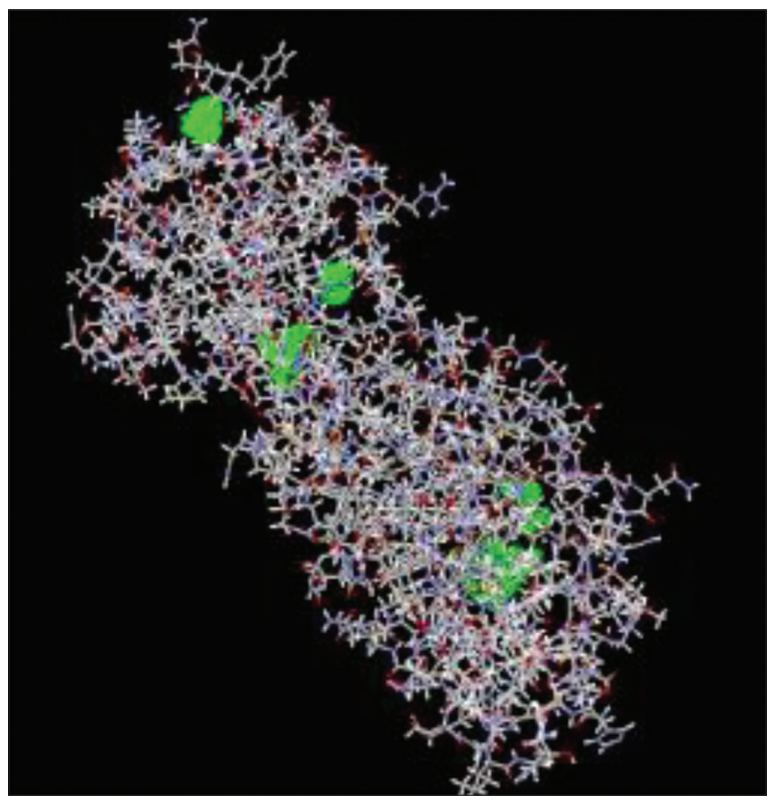

Fig. 2: Representation of identified five cavities in green color in selected pdb 6UL7

\section{RESULTS AND DISCUSSION}

Drug likeness parameters according to Pfizer's rule of five, such as mol. wt, logP, hydrogen bond acceptor, hydrogen bond donors, and no of rotatable bonds of the selected drugs were predicted in silico using pKCSM software, are shown in Table 1, and it was observed that calculated parameters were found within the optimum range. The 3D structures of all selected compounds are also represented in Fig. 1.

Next, in this study, we used that X-ray structure of the COVID-19 main protease in complex with an inhibitor N3 (PDB code 6LU7) was downloaded from protein data bank for molecular docking. The total structure weight of this protein is around 34506.34 DA with 2.1 A resolutions. The molecular docking study was performed using MVD software (https://omictools.com) to investigate the molecular interaction between selected drugs and protease enzyme. The software

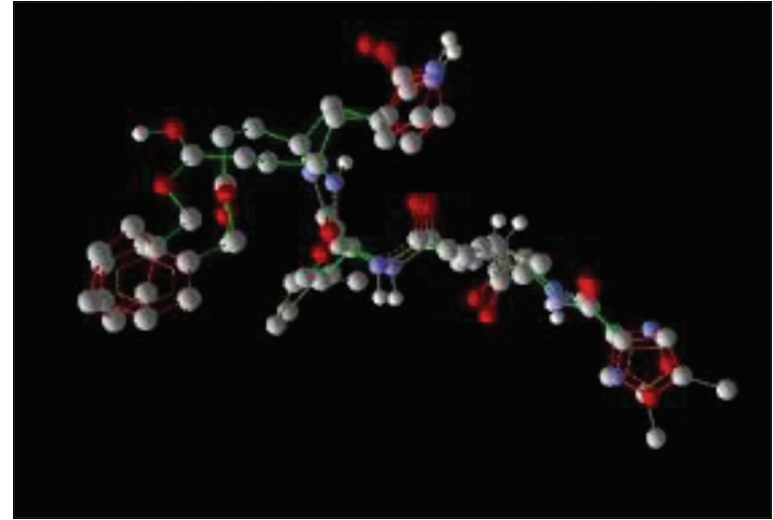

Fig. 3: Superimposed view of the best scoring pose of cocrystallized ligand in pdb $6 \mathrm{UL7}$
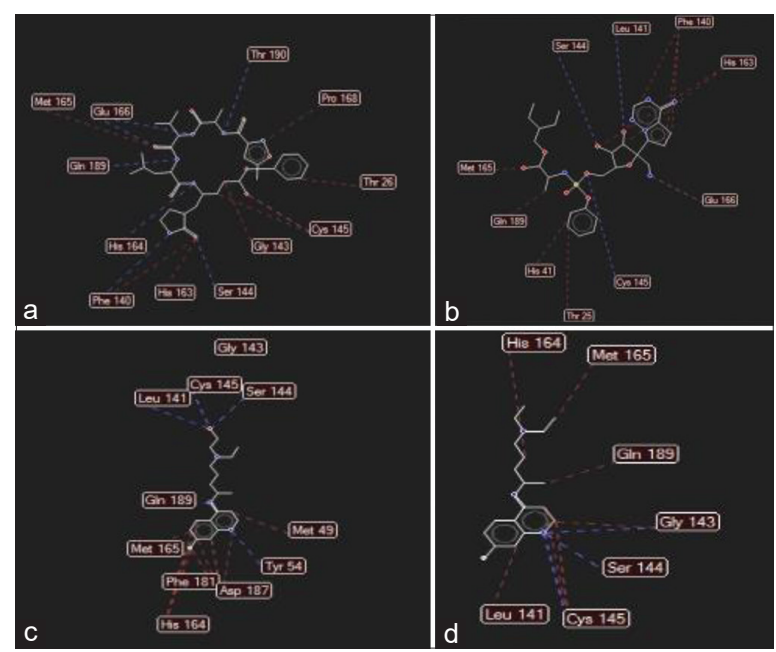

Fig. 4: Docking poses of representative compounds (a) docking pose of cocrystallized ligand; (b) 2D interaction plot of remdesivir; (c) 2D interaction plot of hydroxychloroquine; and (d) 2D interaction plot of chloroquine

Table 1: In silico predicted physicochemical parameter

Name and structure $\quad$ M.Wt. $\quad \log \mathrm{P}^{\mathrm{a}}$ RB $^{\mathrm{b}}$ HBA $^{\mathrm{c}}$ HBD $^{\mathrm{d}} \quad$ PSA $^{\mathrm{e}}$ of compound

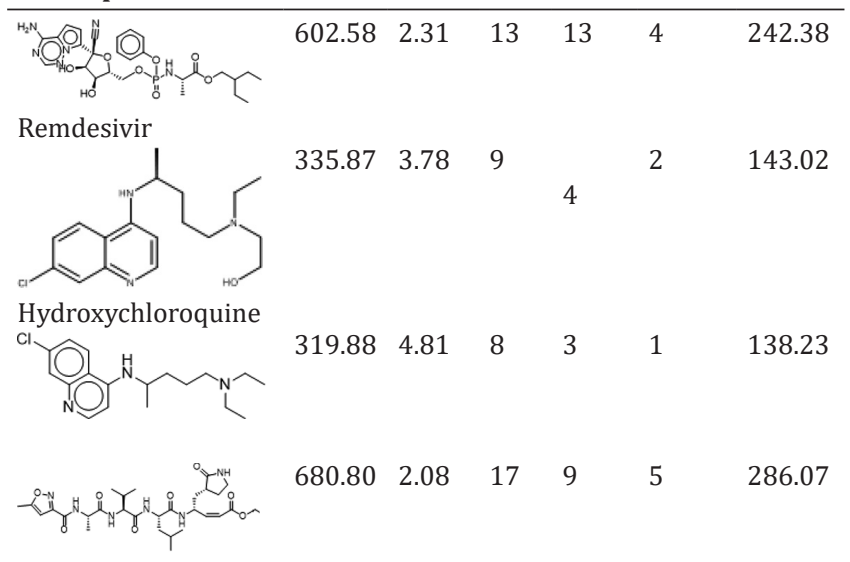

Cocrystallized ligand

HBA: Hydrogen bond acceptor, HBD: Hydrogen bond donors, RB: Rotatable bond

has identified five protected areas of the enzymatic flap, as represented in Fig. 2. The target protein was further refined using protein preparation option, and 3D energy minimized structures of the designed ligands were 
Table 2: The sum of the energies resulting from the interaction of selected drugs and protease enzymes

\begin{tabular}{|c|c|c|c|c|c|}
\hline Ligand & Mol dock score & Rerank score & HBond & Electrostatic bond & Interactions \\
\hline Cocrystallized ligand & -232.04 & -178.63 & -13.22 & 0 & $\begin{array}{l}\text { H Bonding Interactions: Phe 140, Ser 144, His 164, Glu } \\
\text { 166, Gln } 189 \text { and Thr } 190 \\
\text { Steric Interactions: Thr 26, Gly 143, His 163, Met 165, } \\
\text { and Pro } 168 .\end{array}$ \\
\hline Remdesivir & -163.62 & -116.14 & -8.44 & 0 & $\begin{array}{l}\text { H Bonding Interactions: Leu 141, Ser } 144 \text { and Cys } 145 \\
\text { Steric Interactions: Thr 25, His 41, Leu 141, Ser 144, Cys } \\
\text { 145, His } 163 \text {, Met 165, Glu 166, and Gln } 189 .\end{array}$ \\
\hline Hydroxychloroquine & -114.45 & -75.54 & -9.72 & 0 & $\begin{array}{l}\text { H Bonding Interactions: Tyr 54, Leu } 141 \text {, Ser } 144 \text {, Cys } \\
\text { 145, and Gln } 189 \\
\text { Steric Interactions: Met 49, Gly } 143 \text {, His } 164 \text {, Met } 165 \text {, } \\
\text { Phe } 181 \text {, Asp 187, and Gln } 189 .\end{array}$ \\
\hline Chloroquine & -110.25 & -70.44 & -8.14 & 0 & $\begin{array}{l}\text { H Bonding Interactions: Gly } 143 \text {, Ser } 144 \text {, and Cys } 145 \\
\text { Steric Interactions: Leu 141, Gly } 143 \text {, Ser } 144 \text {, Cys } 145 \text {, } \\
\text { His } 163 \text {, His } 164 \text {, Met } 165 \text {, and Gln } 189 .\end{array}$ \\
\hline
\end{tabular}

prepared using Chem Bio Draw 3D Ultra v12.0. The grid was generated using the center coordinates $-10.76,12.64$, and 68.81 in the $\mathrm{x}, \mathrm{y}$, and $\mathrm{z}$ directions at the radius of $10 \AA$, respectively. The validation of the docking protocol was done by overlaying the docked pose of cocrystallized ligand that is N-[(5-MethylIsoxazol-3-YL) Carbonyl]Alanyl-L-Valyl-N-1-((1R,2Z)4-(Benzyloxy)-4-0xo-1-\{[(3R)-2-Oxopyrrolidin-3-YL]Methyl\}But-2Enyl)-L-Leucinamide, as shown in Fig. 3. The cocrystallized has shown interactions with amino acids, namely, Phe 140, Ser 144, His 164, Glu 166, Gln 189, and Thr 190 through forming hydrogen bonding with a moldock score of -232.04. The anti-viral drug remdesivir showed hydrogen bonding with amino acids Leu 141, Ser 144 (via hydroxyl groups of furan ring) and Cys 145 by oxygen attached next to the phosphorous group, respectively. The other stericinteractions with amino acids such as Thr 25, His 41, Leu 141, Ser 144, Cys 145, His 163, Met 165, Glu 166, and Gln 189 were also observed. Next, the most highlighted drug hydroxychloroquine was docked and it exhibited its mol dock score - -114.45 , which was comparable very less to the cocrystallized ligand. The hydrogen bonding interactions with amino acids such as Tyr 54 (through $\mathrm{N}$ of quinoline ring), Leu 141, Ser 144, and Cys 145 by hydroxyl group of aliphatic side chain, respectively were also seen. It have also showed the steric interactions with amino acids, namely, Met 49, Gly 143, His 164, Met 165, Phe 181, Asp 187 , and Gln 189. At last, another molecule of same class chloroquine has shown its mole dock score -110.25 through interacting with Gly 143 , Ser 144, and Cys 145 amino acids by the formation of hydrogen bonding with $\mathrm{N} 1$ of basic quinoline ring. It also exhibited different steric interactions with amino acids Leu 141, Gly 143, Ser 144, Cys 145, His 163, His 164, Met 165, and Gln 189, respectively. The 2D interaction plots of docked poses are represented in Fig. 4 and the results of the molecular docking study on COVID-19 main protease (PDB code 6LU7) are summarized in Table 2, respectively.

\section{CONCLUSION}

By performing the molecular docking study of selected drugs, we are able to understand the interaction between functional groups of these drugs and amino acids of protease enzyme present in the active site. The obtained mol dock score is a representation of hydrogen bonding, steric, and other interactions between enzyme and drugs and plays a crucial role to define enzymatic catalysis. Based on the results of the present study, it can be concluded that these clinical management agents can interfere with the important amino acids in the enzymatic cavity to inhibit the protease enzyme virus. In the future, this study will help the scientist for drug development against COVID-19.

\section{AUTHOR'S CONTRIBUTIONS}

Dr. Pankaj Wadhwa has selected the drugs on the basis of reported literature and also performed the molecular modeling studies using the computational methods. He was also involved in the preparation, reviewing, and editing of the manuscript.

\section{CONFLICTS OF INTEREST}

The author declare no conflicts of interest

\section{AUTHOR'S FUNDING}

Nil.

\section{REFERENCES}

1. Chen H, Guo J, Wang C, Luo F, Yu X, Zhang W, et al. Clinical characteristics and intrauterine vertical transmission potential of COVID-19 infection in nine pregnant women: A retrospective review of medical records. Lancet 2020;395:809-15.

2. Available from: https://www.who.int/docs/default-source/ coronaviruse/situation-reports/20200525-covid-19-sitrep-126. pdf?sfvrsn=887dbd66 2 .

3. Chen Y, Liu Q, Guo D. Emerging coronaviruses: Genome structure, replication, and pathogenesis. J Med Virol 2020;92:418-23

4. Liu X, Zhang B, Jin Z, Yang H, Rao Z. The Crystal Structure of 2019nCoV Main Protease in Complex with an Inhibitor N3. New Jersey: RCSB Protein Data Bank. 2020.

5. John SE, Tomar S, Stauffer SR, Mesecar AD. Targeting zoonotic viruses: Structure-based inhibition of the 3C-like protease from bat coronavirus HKU4--the likely reservoir host to the human coronavirus that causes Middle East Respiratory Syndrome (MERS). Bioorg Med Chem 2015;23:6036-48

6. Gordon CJ, Tchesnokov EP, Feng JY, Porter DP, Götte M. The antiviral compound remdesivir potently inhibits RNA-dependent RNA polymerase from Middle East respiratory syndrome coronavirus. J Biol Chem 2020;295:4773-9

7. Colson P, Rolain JM, Lagier JC, Brouqui P, Raoult D. Chloroquine and hydroxychloroquine as available weapons to fight COVID-19. Int J Antimicrob Agents 2020;55:105932.

8. Pires DE, Blundell TL, Ascher DB. pkCSM: Predicting small-molecule pharmacokinetic and toxicity properties using graph-based signatures. J Med Chem 2015;58:4066-72.

9. Shaghaghi N. Molecular docking study of novel COVID-19 protease with low risk terpenoides compounds of plants. ChemRxiv 2020;10:1-9. 